\title{
A COMPARISON OF RESISTED AND ASSISTED SPRINT TRAINING IN COLLEGIATE SPRINTERS
}

\author{
Murray, J. ${ }^{1}$, Harris, C. ${ }^{2}$, \\ Adams, K. J. ${ }^{3}$, Joseph Berning, J. ${ }^{4}$, \\ DeBeliso, M. ${ }^{{ }^{*} i}$ \\ ${ }^{1}$ Utah State University, Logan UT, USA \\ ${ }^{2}$ Metropolitan State University of Denver, Denver, CO, USA \\ ${ }^{3}$ California State University Monterey Bay, Seaside, CA, USA \\ ${ }^{4}$ New Mexico State University, Las Cruces, NM, USA \\ ${ }^{5}$ Southern Utah University, Cedar City, UT, USA
}

\begin{abstract}
:
Sprint time (ST) is the product of stride length (SL) and stride rate (SR). Increases in either of these variables results in speed improvement. Purpose: To compare resisted (RST) and assisted sprint training (AST) on sprint performance. Methods: Twenty (10 male, 10 female) collegiate sprinters and hurdlers were randomly divided into two training groups: RST (age: $21.8 \pm 1.8 \mathrm{yrs}$, height: $1.73 \pm 0.10 \mathrm{~m}$, mass: $69.5 \pm 12.8 \mathrm{~kg}$ ) and AST (age: $22.2 \pm 2.4 y r s$, height: $1.76 \pm 0.10 \mathrm{~m}$, mass: $69.1 \pm 9.9 \mathrm{~kg}$ ). Each group trained 3 days/week for 6 weeks. The RST group used a combination of weighted sled pulls, uphill sprinting and depth jumps. The AST group combined downhill running, towing, sprint ladders and single leg bounds. Prior to and following the training interventions SL and ST (10 meter sprint time) were recorded at sprint distances of $30 \mathrm{~m}, 60 \mathrm{~m}$, and $120 \mathrm{~m}$ during the last 10 meters of each sprint distance. Pre-post training ST and SL were compared within training groups at each sprint distance using paired t-tests. Additionally, a gain score was calculated by taking the difference between the post and pre test scores. The gain scores for SL and ST were compared between training groups via independent t-tests at each sprint distance. Results: For the RST 30m, ST $(1.18 \pm 0.08 \mathrm{~s}$ vs $1.14 \pm 0.08 \mathrm{~s}, \mathrm{p}<0.01)$ and SL $(2.06 \pm 0.08 \mathrm{~m}$ vs. $2.09 \pm 1.14 \mathrm{~m}, \mathrm{p}<0.01)$ were significantly improved. For the AST, 30m ST $(1.19 \pm 0.08 \mathrm{~s}$ vs. $1.18 \pm 0.08 \mathrm{~s}, \mathrm{p}<0.01)$ and SL $(2.10 \pm 0.13 \mathrm{~m}$


vs. $2.11 \pm 0.13, \mathrm{p}=0.04)$ were significantly improved. No improvements in ST or SL were detected for either the RST or AST groups at the $60 \mathrm{~m}$ sprint distance. For the RST 120m, ST $(1.13 \pm 0.07 \mathrm{~s}$ vs. $1.12 \pm 0.08 \mathrm{~s}, \mathrm{p}<0.01)$ was significantly improved. For the AST $120 \mathrm{~m}, \mathrm{ST}$ $(1.12 \pm 0.07 \mathrm{~s}$ vs. $1.11 \pm 0.07 \mathrm{~s}, \mathrm{p}<0.01)$ and SL $(2.30 \pm 0.08$ vs. $2.33 \pm 0.08, \mathrm{p}<0.01)$ were significantly improved. At the 30M interval, the RST demonstrated significant improvements in SL and ST as compared to the AST $(\mathrm{p}<0.01)$. However, at the $120 \mathrm{M}$ interval, the AST demonstrated a significant improvement in SL as compared to the RST ( $p<0.01$ ). Conclusion: Within the parameters of this study, RST and AST methods have proven effective at improving sprint performance. It appears that RST protocols may be of greatest benefit for improving sprint performance at shorter distances, while AST protocols may be of greater benefit at longer sprinting distances.

Keywords: assisted, resisted, sprint, collegiate, stride rate, stride length

\section{Introduction}

Speed is a function of stride length multiplied by stride rate ( $S=S L \times S R$ ). To improve running speed through training, the training mechanism should improve SL, SR, or both. Typically, training mechanisms to improve sprinting speed involve some type of resisted sprint training (RST) where sprinting is done against a resistance. RST is done to impact leg strength and power and subsequently, improve stride length. Another approach to sprint training involves training at supra-maximal speeds, where the training mechanism is assisting the runner or Assisted Sprint Training (AST). RST in the form of sled pulling has resulted in improvements in sprinting speed (Bachero-Mena \& González-Badillo, 2014: Jakeman, McMullan, \& Babraj, 2016; Kawamori, Newton, Hori, \& Nosaka, 2014; Lockie, Murphy, \& Spinks, 2003; Makaruk, Sozański, Makaruk, \& Sacewicz, 2013; Paradisis \& Cooke, 2006; Upton, 2011; West, Cunningham, Bracken, Bevan, Crewther, Cook, \& Kilduff, 2013) and SL (Kawamori, Newton, Hori, \& Nosaka, 2014; Lockie, Murphy, \& Spinks, 2003; Makaruk, Sozański, Makaruk, \& Sacewicz, 2013; West, Cunningham, Bracken, Bevan, Crewther, Cook, \& Kilduff, 2013). RST using uphill sprint training also resulted in increased sprinting speed (Jakeman, McMullan, \& Babraj, 2016) as has plyometric training (Lockie, Murphy, \& Spinks, 2003). For AST, training using both towing (Upton, 2011) and downhill running (Paradisis \& Cooke, 2006) has resulted in speed increases. Most studies have focused on one or few training modalities for either RST or AST (Bachero-Mena \& González-Badillo, 2014: Jakeman, McMullan, \& Babraj, 2016; Kawamori, Newton, Hori, \& Nosaka, 2014; Lockie, Murphy, \& Spinks, 2003; Makaruk, Sozański, Makaruk, \& Sacewicz, 2013; Paradisis \& Cooke, 
2006; Upton, 2011; West, Cunningham, Bracken, Bevan, Crewther, Cook, \& Kilduff, 2013).

Weight sleds are a very common training apparatus used to build power and explosiveness in many sports and are a form of RST. A 2011 study by Martinopoulou et al. found that sprint - speed training with pull against resistance results in running velocity improvement by increasing stride length (SL). This is based on the fact that resistance helps increase the power output of the hip and knee joint muscle groups which further increases thrust and SL, a prerequisite to improve speed in the acceleration phase. Another form of RST is uphill sprinting. In a study conducted by Kunz \& Kaufmann (1981) it was found that running velocity was slower than that of level ground running $(8.35 \mathrm{~m} / \mathrm{s}$ to $8.85 \mathrm{~m} / \mathrm{s})$ and that the subjects had shorter stride lengths and longer ground contact times. Kunz \& Kaufmann believed that uphill sprinting will increase the stress placed on the hip extensor muscle groups as the athlete will attempt to maximize stride length, therefore increasing this component (SL) on the flat surface. Kunz and Kaufmann noted that an incline of greater than 3\% would be beneficial in developing the forceful hip extensor movements required to achieve desired improvements in stride length. In this exercise, the athlete started from a crouch position and sprinted 100 meters up a hill.

Previous research indicates that downhill running, sprint ladders, towing, and single leg bounding are forms of AST. According to Ross (2005), SR is determined mostly by two main factors; ground contact time and swing time. Of these, Ross states that ground contact time is far more important because stride rates become faster by the amount of time not spent on the ground. Swing time can be trained and improved but the greatest result comes from shorter ground contact times. Single leg bounding is a drill used to create power and explosiveness in sprinters. Landing on the same leg required the athlete to build strength and balance as well as help them learn to react quickly once they hit the ground. The focus of this drill is not on covering extreme distances but in building quick reaction off the ground thus reducing the amount of time in contact with the ground on each jump. Downhill sprinting has also been shown to be an effective training tool for improved SR. Maximum running speed and step rate were increased significantly $(\mathrm{p}<0.05)$ in a 35-m running test after downhill training in a study performed by Paradisis and Cooke (2006). Downhill running is an AST form of sprint training using a hill of at least $3 \%$ grade. This over speed training results in improved SR and overall running speed, the hope is that muscle memory will allow the athlete to take these gains to the flat running surface. Towing is a common form of assisted sprinting used quite often in track and field. In a study conducted by Gervais and LeBlanc (2004), ground support time was found to be significantly shorter in assisted sprinting. In this drill, the athlete was harnessed to a pulley system that was 
attached to another athlete standing 10 meters in front of the harnessed athlete. As the front athlete accelerated the athlete, being towed was forced by the pulley system to accelerate at a faster rate than the first athlete in order to keep up. This version of overspeed training allows the athlete to train at speed intensities above what is achievable independently.

The purpose of this study was to compare Resisted Sprint Training using a combination of RST modalities with Assisted Sprint Training using multiple AST modalities relative to sprint performance and kinematics (SR and SL). It was hypothesized that both training modalities would improvements in sprint speed and that the AST would lead to superior improvements in SR while RST would lead to greater gains in SL.

\section{Methods}

\subsection{Participants}

The participants for this study were a convenience sample of male and female collegiate track and field athletes that compete in the sprint and hurdle events at the NCAA Division I level. Permission from the head coach was granted prior to the study. Student athletes were asked to volunteer for the study. Permission from the Institutional Review Board was obtained before conducting any training or assessments of the participants. Prior to engaging in any testing each of the participants signed an informed consent form that had been previously approved by the Institutional Review Board of Southern Utah University.

\subsection{Instruments and Apparatus}

The study training and testing sessions were conducted at the Athletic Complex at Southern Utah University (Figure 1). Equipment necessary to conduct this study included Accusplit 601X hand held stopwatches (Accusplit, Pleasanton, California, USA), a standard video camera, and a running track. The running track was marked in three separate 10 meter zones in increments 10 centimeters. The first was a 30 meter sprint, with the final 10 meters timed and measured for SL. The second was a 60 meter sprint, with the final 10 meters timed and SL measured. Finally, the participants performed a 120 meter sprint with the final 10 meters timed and SL measured. 


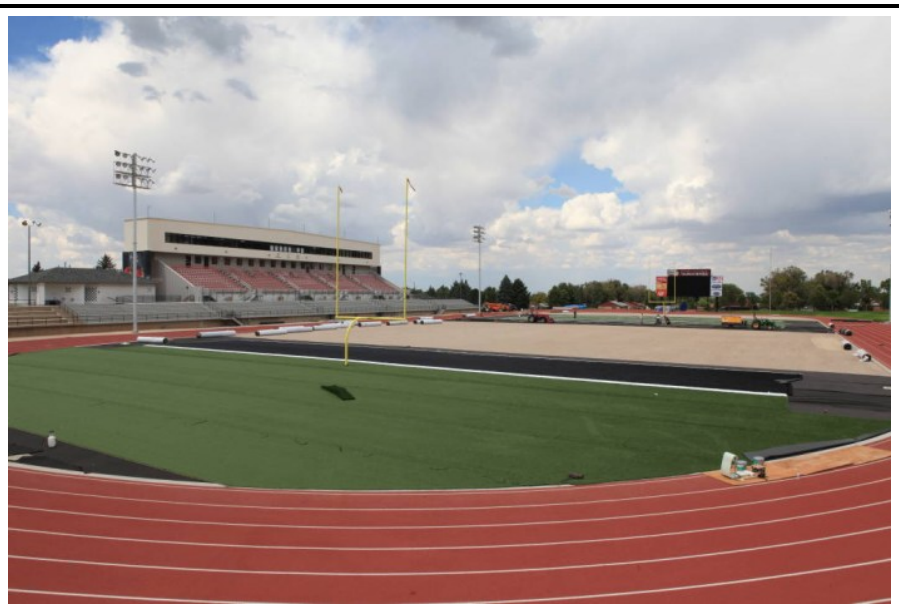

Figure 1: Southern Utah University Athletics Facility

\subsection{Procedures}

The study initiated with a briefing session to address any comments or concerns posed by the participants. Next age, height and weight were recorded. Pre training assessment of the dependent variables was then conducted as described below (see Protocol for Assessing SR, SL, and Running Speed). The pre training assessment was conducted during the pre-competitive training phase ensuring the participants were properly conditioned. Following the pre training assessment session, the experimental groups (RST and AST) were constructed using a stratified random process assuring both experimental groups were balanced for gender (5-female and 5-male per experimental group).

Once divided into one of two experimental training groups the athletes performed training techniques thought to be beneficial to one of two areas, stride length (SL), and stride frequency (SR). Drills for SL included sleds pulling, running uphill, and depth jumps. For the purposes of this study, the sleds were weighted with $20 \%$ of the athlete's body weight to insure an equal work load for all involved and to allow the athlete to maintain correct running posture throughout the exercise. The sled was attached to the athlete using a harness around their waist. The athlete performed a series of 20 meter sprints, with the focus on keeping their center of mass forward in order to move the sled as quickly as possible. For the purposes of this study, a hill with a grade of $6 \%$ was chosen. The athletes were required to drive off the ground with extreme force in order to accelerate up the hill at the desired velocity. This exercise was used as a focus of training for stride length and was performed 3 times over the 100 meter distance. For the purpose of this study plyometric training involving depth jumps from a platform was implemented. Dropping from a platform at a height of $40.6 \mathrm{cms}$ ( 16 inches) allowed the athlete to land recover and explode forward horizontally. This drill was performed only 10 times per training session as it is a ballistic drill involving extreme forces and change of direction. The goal is to drop off the box and immediately 
upon making contact with the ground change direction and jump forward horizontally as far as possible.

Drills used for SR included downhill running, towing on a cable/pulley system, use of a sprint ladder and bounding. The following independent variables were all used for stride frequency training. They included downhill running, sprint ladders, towing, and single leg bounding. Downhill running was conducted using a hill of at $\approx 3 \%$ grade for a distance of 50 meters (6 sets, total of 300 meters). In the towing drill, the athlete was harnessed to a pulley system that was attached to another athlete standing 10 meters in front of the harnessed athlete. As the front athlete accelerated the athlete being towed was forced by the pulley system to accelerate at a faster rate than the first athlete in order to keep up. This drill was performed five times over a distance of 30 meters. This version of over-speed training allows the athlete to train at intensities above what is achievable independently. The AST group also performed sprint ladders to aid their training for stride frequency. Sprint ladders are a series of markers set up at a predetermined length based off the height of each athlete. Each stride was increased in length over 10 strides at which point the athlete worked to maintain the same stride length while increasing their turnover rate for the next 10 strides. This drill allowed the athlete to increase their stride frequency without increasing their stride length over the final 10 strides. Single leg bounding is a drill used to create power and explosiveness in sprinters. The athlete performed 10 bounds using the same leg. Landing on the same leg required the athlete to build strength and balance as well as help them learn to react quickly once they hit the ground. The focus of this drill is not on covering extreme distances as in the depth jumps but in building quick reaction off the ground thus reducing the amount of time in contact with the ground on each jump.

The intervention period of the study lasted 6 weeks. Prior to each training and assessment session, a dynamic warm-up was performed as stated below. Each group also continued with their regularly scheduled training program including, strength training, interval training, and technical work (held constant between experimental groups). Figure 2 represents the specific training program for each experimental group (modality, frequency, and intensity). The PI was the participant's track coach and was present for all training sessions and conducted the pre and post study DV assessments.

\subsection{Dynamic WU}

The DYN WU took approximately 10-15 minutes to complete and consisted of a series of jogging movements (total 1000 meters) that included: 
Skips;

High Knees;

Butt kicks;

Carioca Left;

Carioca Right;

\section{Lunges;}

Walking tall;

Run Backward, and

Form Run 200 meters.

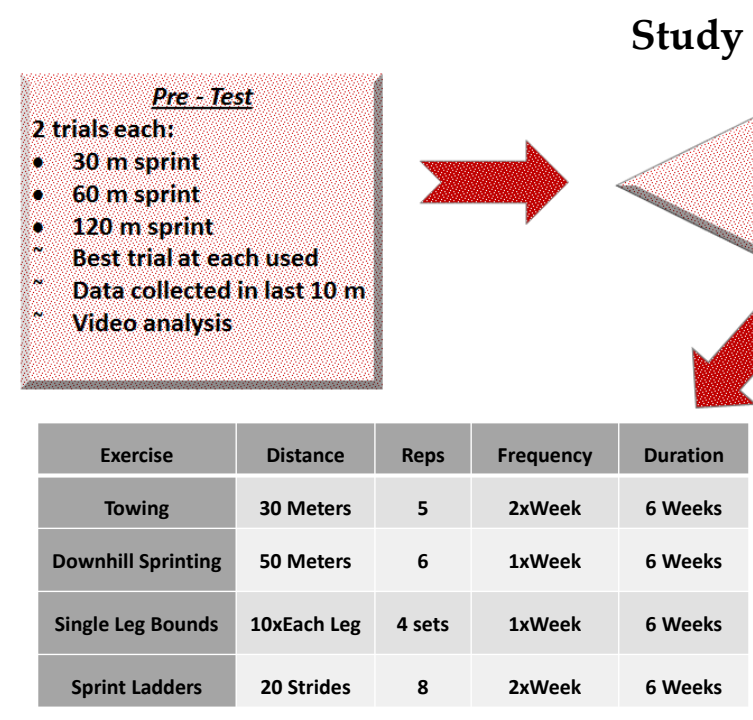

AST Protocol

\section{Study Flow Diagram}

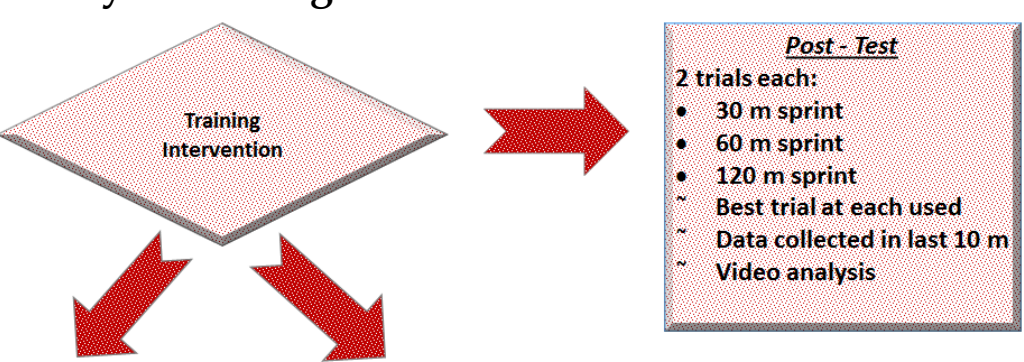

Figure 2: Study time line of events and procedures

Note: Sled, 20\% body mass; Uphill Grade, $6 \%$.

\subsection{Protocol for Assessing SR, SL, and Running Speed}

Prior to training each athlete participated in a pre-test to determine their stride frequency, stride length and running speed for sprint distances of 30, 60, and 120 meters (M). The researcher used a stop watch to time the participants running speed in each of three sprint distances during the last 10M. SR and SL were assessed during the final $10 \mathrm{M}$ of each of the three sprint distances. These measurements were made with the athlete running over a section of track that was $10 \mathrm{M}$ long and marked every 10 centimeters. This $10 \mathrm{M}$ section of the track was videoed from the sagittal plane for the assessment of SR and SL. SL was measured from toe contact of one limb to the next successive toe contact of the same limb. SR was determined using the formula SR=S/SL. Two testing trials were performed at each distance with the fastest time for each trial being used for analysis. All of the sprint trials were conducted during the same day in sequential fashion (i.e. 30M followed, $60 \mathrm{M}$, and finally $120 \mathrm{M}$ ) separated by $5-10$ minutes. The pre and post-test assessments followed the same order and procedures to ensure accuracy in testing and familiarity for the participants. 


\subsection{Reliability}

The reliability of short sprints has been reported to range from r=0.89-0.97 (Miller, 2012). The reliability of handheld timing devices has also been reported to range from ICC $=0.90-0.97$. The concurrent validity between electronic and hand held devices is considered very high (ICC=0.98) (Mayhew et al., 2010). Sagittal plane video analysis of running mechanics such as foot strike patterns is considered highly reliable (Bertelsen, Jensen, Nielsen, Nielsen, \& Rasmussen, 2013).

\subsection{Statistical Analysis}

The dependent variables (DVs) in this study were running speed, SR and SL at sprint distances of 30, 60, and 120 meters. The DVs were compared within each experimental group (RST, AST) with paired t-tests. A gain score was calculated for each DV (post-pre score) and compared between experimental groups with an independent t-test. For the purpose of statistical significance alpha was $\leq 0.05$.

\section{Results}

Twenty collegiate track athletes (sprinters and hurdlers) participated in this study where each experimental group (AST and RST) was comprised of 5-males and fivefemales. Table 1 provides participant descriptive information of height, body mass and age.

Table 1: Participant descriptive information

\begin{tabular}{|c|c|c|c|c|}
\hline & N & Age (years) & Height (cms) & Mass (kgs) \\
\hline RST & 10 & $21.8 \pm 1.8$ & $173.0 \pm 10.0$ & $69.5 \pm 12.8$ \\
\hline AST & 10 & $22.2 \pm 2.4$ & $176.0 \pm 10.0$ & $69.0 \pm 9.9$ \\
\hline
\end{tabular}

Participant mean and standard deviations for descriptive information. 
Table 2: RST and AST: Speed, Stride Length and Stride Rate Pre and

Post Intervention Assessment
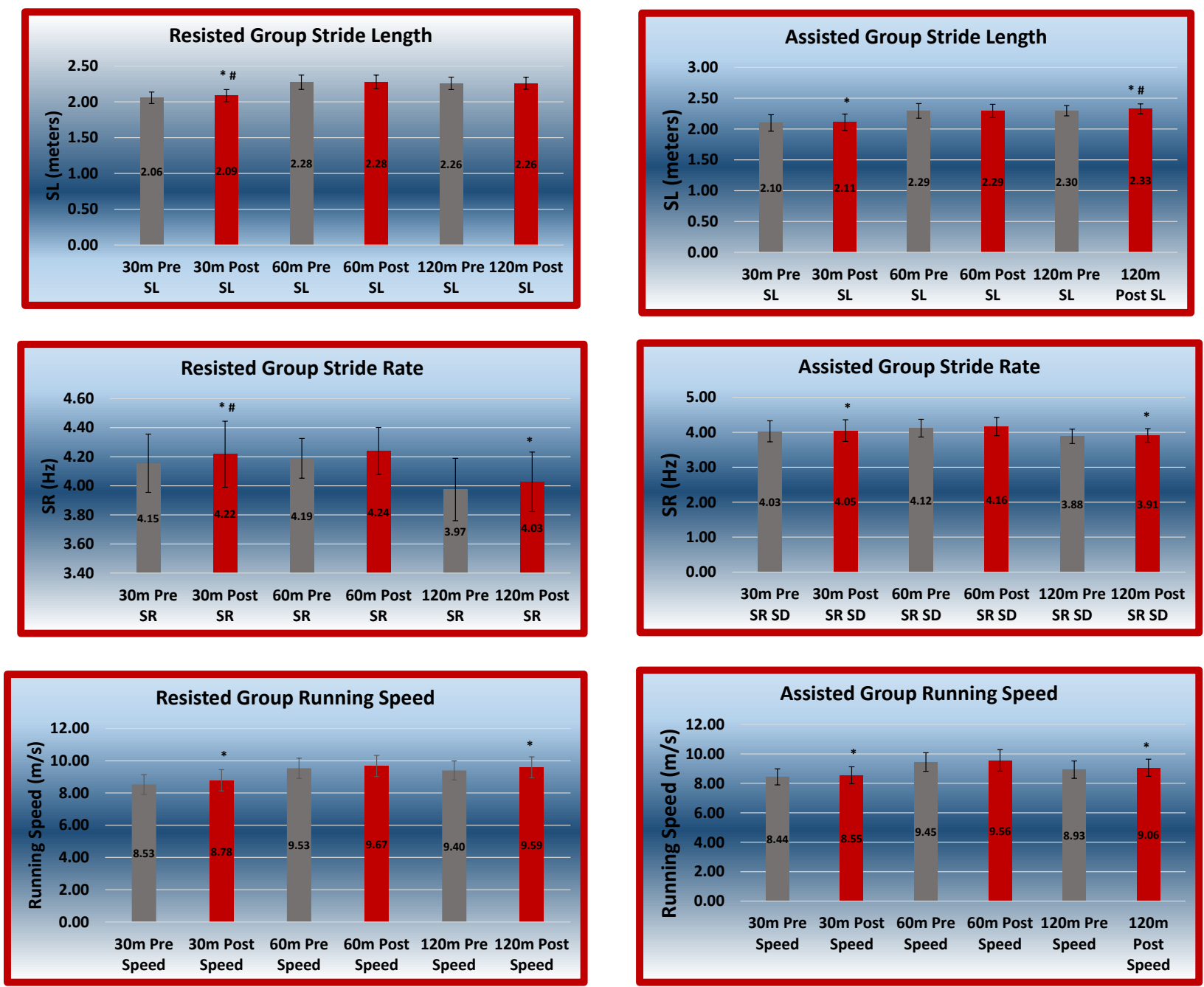

Speed-meters/seconds, SR-stride rate (Hz), SL-stride length (meters). *significant differences pre-post within groups $\mathrm{p}<0.05$; \#-significant difference in gain score between groups $\mathrm{p}<0.05$.

Table 2 provides pre and post DV assessment data for both the AST and RST experimental groups. Significance improvements $(p<0.05)$ for the DVs within the AST were as follows:

- SL over 30M;

- SR over 30M;

- SL over 120M;

- SR over $120 \mathrm{M}$;

- 10 meter time (running speed) over 30M;

- 10 meter time (running speed) over $120 \mathrm{M}$.

Significance improvements $(\mathrm{p}<0.05)$ for the DVs within the RST were as follows:

- SL over 30M;

- SR over 30M;

- $\quad$ SR over $120 \mathrm{M}$; 
- 10 meter time (running speed) over 30M;

- 10 meter time (running speed) over 120M.

Significance improvements $(\mathrm{p}<0.05)$ for the DVs gains scores were found between the RST and AST group were as follows:

- SL over 30M (Greater for RST);

- SL over 120M (Greater for AST).

\section{Discussion}

The main findings in this study included an increase in running speed at 30 meter and 120 meters following both the resisted and assisted sprint training. For distances similar to the 30-meter measurement in our study, increases in running speed have been reported following RST (Bachero-Mena \& González-Badillo, 2014: Paradisis \& Cooke, 2006; Upton, 2011; West, Cunningham, Bracken, Bevan, Crewther, Cook, \& Kilduff, 2013). Additionally, running speed increases as a result of RST were reported for shorter sprint distances, ranging from five meters to just over 20 meters (Jakeman, McMullan, \& Babraj, 2016; Kawamori, Newton, Hori, \& Nosaka, 2014; Lockie, Murphy, \& Spinks, 2003; Makaruk, Sozański, Makaruk, \& Sacewicz, 2013). Our study was unique, demonstrating running speed improvements for longer distance sprinting (up to 120 meters). The previously reported speed increases with RST training have come using applications of individual resisted training modalities of sled pulling, uphill running and plyometrics. Our study combined these modalities in the RST group. Speed increases following AST using either downhill running (Paradisis \& Cooke, 2006;) or a combination of downhill running and towing (Upton, 2011) have been reported for distances of 35 meters. Again, we were able to demonstrate speed increases up to 120 meters.

Another finding of our study was changes in stride kinematics following both RST and AST. For the RST group SL increased at 30 meters. This was consistent with previous findings for that distance (West, Cunningham, Bracken, Bevan, Crewther, Cook, \& Kilduff, 2013). Former studies also have demonstrated SL increases for distances of five meters to 20 meters (Kawamori, Newton, Hori, \& Nosaka, 2014; Lockie, Murphy, \& Spinks, 2003; Makaruk, Sozański, Makaruk, \& Sacewicz, 2013; West, Cunningham, Bracken, Bevan, Crewther, Cook, \& Kilduff, 2013). Following RST, SR increased at 30 and 120 meters. Previous studies have been equivocal, showing decreased SR following RST (Makaruk, Sozański, Makaruk, \& Sacewicz, 2013) and increased SR following a combination of RST and AST training (Paradisis \& Cooke, 2006). For our AST group, SL and SR increased at both 30 meters and 120 meters. 
Additionally, AST was found to produce superior results over RST for SL at both 30 meters and 120 meter.

Another unique aspect of our study was the use of collegiate (NCAA Division I) athletes specifically trained for sprinting and hurdling. Some previous studies have used collegiate (Upton, 2011) semi-professional (Jakeman, McMullan, \& Babraj, 2016) or professional (West, Cunningham, Bracken, Bevan, Crewther, Cook, \& Kilduff, 2013) athletes. However, these were team sport athletes, not track and field sprint athletes. Many of the prior studies have used "physically active" subjects (Jakeman, McMullan, \& Babraj, 2016; Kawamori, Newton, Hori, \& Nosaka, 2014; Lockie, Murphy, \& Spinks, 2003; Makaruk, Sozański, Makaruk, \& Sacewicz, 2013; Paradisis \& Cooke, 2006). The results of the current study are noteworthy, considering the history of sprint training specificity with this group. Future studies may benefit from examining both a combined training approach, as was use currently and individual resisted and assisted modalities to help delineate the efficacy of the different modalities.

The main assumption in this study is that all participants put forth a maximum effort during both the pre and post-testing. The time of season that this testing took place played a large part in effort. The pre-test was taken during the pre-competitive phase of training, immediately preceding the participant's main competitive season. This was done to ensure that the participants were in good physical condition, as well as motivated to perform well on the testing. The post-testing took place one week before the outdoor conference championship meet, also ensuring that the participants were motivated and in peak condition. The timing of the post-test also allowed for those participants who did not travel to the conference championship meet to take place in the testing.

As with most studies that use competitive athletes, a limitation in this study was that of sample size. The research was limited to just one collegiate track and field team. This is admittedly a very small sample of the 300 plus universities in the country. However, it would have proved to be extremely difficult to convince other collegiate coaches to alter their training regimens in order to fit the research protocols of this study. The second limitation was the age of the subjects participating in the study (age range of 18-26). While this limitation could be considered an issue, the majority of collegiate track and field athletes fall directly into this same age bracket. This is not to say that these results would not be beneficial to high school age athletes or even post collegiate competitors

While this study has shown how valuable RST and AT can be to improved sprint success, there is no way to know which training variable used within each experimental training group (RST or AST) was most responsible for the gains made. Further studies would need to be done comparing the specific improvements from each of the resisted 
training variables (weighted sleds, uphill sprinting and depth jumps) as well as the assisted training variables (towing, downhill sprinting, single leg bounding and sprint ladders) in order to provide a more precise measure of the effects of each variable independently.

\section{Practical Application}

This study found a significant impact of RST and AST with regards to improving SL at specific distances, as well as improved 10 meter sprint times over specific distances. Similar training used for the express purpose of improving stride length or 10 meter sprint times may also elicit the desired results. These training methods, used along with a properly designed periodized resistance training program, will most likely bring about positive results in the college age track and field sprint athlete. Comparable results might be expected if a similarly designed training program were used for high school aged athletes as well as post-collegiate or elite athletes.

\section{Acknowledgements}

The author would like to thank the participating members of the Southern Utah University Men's and Women's Track and Field teams for their cooperation with this study.

\section{Conflict of Interest Declaration}

No funding was received for this research. The authors have no conflict of interest related to this research.

\section{References}

1. Bachero-Mena, B., \& González-Badillo, J. J. (2014). Effects of resisted sprint training on acceleration with three different loads accounting for 5, 12.5, and 20\% of body mass. Journal of Strength E Conditioning Research, 28(10), 2954-2960.

2. Bertelsen, M. L., Jensen, J. F., Nielsen, M. H., Nielsen, R. O., \& Rasmussen, S. (2013). Footstrike patterns among novice runners wearing a conventional, neutral running shoe. Gait $\mathcal{E}$ posture, 38(2), 354-356.

3. Jakeman, J. R., McMullan, J., \& Babraj, J. A. (2016). Efficacy of a four-week uphill sprint training intervention in field hockey players. Journal of Strength $\mathcal{E}$ Conditioning Research, 30(10), 2761-2766. 
4. Kawamori, N., Newton, R. U., Hori, N., \& Nosaka, K. (2014). Effects of weighted sled towing with heavy versus light load on sprint acceleration ability. Journal of Strength \& Conditioning Research, 28(10), 2738-2745.

5. Kunz, H., \& Kaufmann, D. (1981). Biomechanics of hill sprinting. Track Technique, 82, 2603-2605.

6. Lockie, R. G., Murphy, A. J., \& Spinks, C. D. (2003). Effects of resisted sled towing on sprint kinematics in field-sport athletes. Journal of Strength $\mathcal{E}$ Conditioning Research, 17(4), 760-767.

7. Makaruk, B., Sozański, H., Makaruk, H., \& Sacewicz, T. (2013). The effects of resisted sprint training on speed performance in women. Human Movement, 14(2), 116-122.

8. Martinopulou, K., Argeitaki, P., Pardisis, G., Katsikas, C., \& Smirniotou, A. (2011). The effects of resisted training using parachute on sprint performance. Biology of Exercise Science, 7(1).

9. Mayhew, J.L., Houser, J.J., Briney, B.B., Williams, T.B., Piper, F.C., \& Brechue, W.F. (2010). Comparison between hand and electronic timing of 40-yd dash performance in college football players. Journal of Strength and Conditioning Research, 24(2), 447-451.

10. Miller, T. (2012). NSCA's Guide to Tests and Assessments. Human Kinetics, Champaign, IL, USA.

11. Paradisis, G. P., \& Cooke, C. B. (2006). The effects of sprint running training on sloping surfaces. Journal of Strength $\mathcal{E}$ Conditioning Research, 20(4), 767-777.

12. Ross, B. (2005). Breakthrough training for breakaway running. Retrieved from http://www.bearpowered.com

13. Upton, D. E. (2011). The effect of assisted and resisted sprint training on acceleration and velocity in Division IA female soccer athletes. Journal of Strength $\mathcal{E}$ Conditioning Research, 25(10), 2645-2652.

14. West, D. J., Cunningham, D. J., Bracken, R. M., Bevan, H. R., Crewther, B. T., Cook, C. J., \& Kilduff, L. P. (2013). Effects of resisted sprint training on acceleration in professional rugby union players. Journal of Strength $\mathcal{E}$ Conditioning Research, 27(4), 1014-1018. 
Murray, J., Harris, C., Adams, K. J., Joseph Berning, J., DeBeliso, M.

A COMPARISON OF RESISTED AND ASSISTED SPRINT TRAINING IN COLLEGIATE SPRINTERS

Creative Commons licensing terms

Authors will retain the copyright of their published articles agreeing that a Creative Commons Attribution 4.0 International License (CC BY 4.0) terms will be applied to their work. Under the terms of this license, no permission is required from the author(s) or publisher for members of the community to copy, distribute, transmit or adapt the article content, providing a proper, prominent and unambiguous attribution to the authors in a manner that makes clear that the materials are being reused under permission of a Creative Commons License. Views, opinions and conclusions expressed in this research article are views, opinions and conclusions of the author(s). Open Access Publishing Group and European Journal of Physical Education and Sport Science shall not be responsible or answerable for any loss, damage or liability caused in relation to/arising out of conflict of interests, copyright violations and inappropriate or inaccurate use of any kind content related or integrated on the research work. All the published works are meeting the Open Access Publishing requirements and can be freely accessed, shared, modified, distributed and used in educational, commercial and noncommercial purposes under a Creative Commons Attribution 4.0 International License (CC BY 4.0). 